\title{
Assessing the Temporal and Spatial Dynamics of the Dengue Epidemic in Northern Sri Lanka using Remote Sensing Data, GIS and Statistical Analysis
}

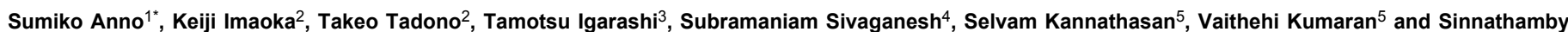
Noble Surendran 5

${ }^{1}$ Shibaura Institute of Technology, Tokyo, Japan

2 Japan Aerospace Exploration Agency, Ibaraki, Japan

${ }^{3}$ Remote Sensing Technology Center of Japan, Tokyo, Japan

${ }^{4}$ Regional Epidemiologist, Jaffna, Sri Lanka

5 University of Jaffna, Jaffna, Sri Lanka

*Corresponding author: Sumiko Anno, Shibaura Institute of Technology, Tokyo, Japan, Tel: 81-3-5859-8363; Fax: 81-3-5859-8401; E-mail: annou@sic.shibaurait.ac.jp

Received date: Oct 30, 2014, Accepted date: Nov 24, 2014, Published date: Nov 28, 2014

Copyright: (c) 2014 Anno S, et al. This is an open-access article distributed under the terms of the Creative Commons Attribution License, which permits unrestricted use, distribution, and reproduction in any medium, provided the original author and source are credited.

\begin{abstract}
Dengue outbreaks are affected by biological, ecological, socio-economic and demographic factors that vary with time and space. These spatial and temporal variables have been examined separately with some success, but still elude systematic understanding. The present study investigates the covariance of spatial and temporal factors for dengue outbreaks in the northern region of Sri Lanka. The relations identified herein demonstrate spatio-temporal dynamics of the disease and can inform surveillance and control strategies. Multi-satellite remote sensing (RS) data were used to construct an index comprising rainfall, humidity and temperature data. RS data gathered by ALOS/ AVNIR-2 and a digital land cover map were used to extract land usage information. Other data on relevant factors and dengue outbreaks were collected through institutions and public databases. RS and other data were integrated and analysed for spatial association analysis and spatial statistics. Our findings show that a combination of ecological, socio-economic and demographic factors can predict spatial and temporal trends in dengue outbreaks.
\end{abstract}

Keywords: Dengue; Ecological, socio-economic and demographic factors; Local Moran LISA statistics; Temporal analysis; Spatial association analysis; Spatial statistical analysis

\section{Introduction}

Since the early 1960s, dengue fever has emerged as a major vectorborne viral disease and a significant source of the childhood fever burden in Sri Lanka. Sri Lankan health officials reported 44,456 dengue cases in 2012, corresponding to a rate of 220 infections per 100,000 individuals. Approximately a quarter of reported cases occurred in children under 15 years of age [1]. Dengue is now considered to be hyper endemic to Sri Lanka, with detected cocirculation of multiple serotypes $[2,3]$.

Dengue outbreaks depend on ecological, socio-economic and demographic factors that vary with time and space. Disease-promoting factors include:

1) Climate variables of rainfall, humidity and temperature [4];

2) Changes in land cover, particularly rapid unplanned expansion of urban areas with inadequate housing and infrastructure [5-11]; and

3) High population density [12].

Standing water from rainfall occupies natural depressions (wetlands, streams, etc.) and human-made containers to create a high surface area of mosquito habitat. High levels humidity also increases mosquito fecundity. Temperature increases impact virus development and vector survival, leading to increases in the proportion of infectious vectors, mosquito range and bite rates. As the time required for a virus to become infectious decreases, the virus spreads more efficiently [4].

Rapid unplanned expansion of urban areas leads to development of communities with inadequate water, sewer and waste management. The lack of an adequate municipal water supply in such urban areas often leads residents to store water in large open containers, such as clay jars and cisterns. Automobile tires, plastic containers and other forms of debris that build up in crowded urban environments create a high surface area of standing water [7-10] which becomes mosquito habitat. Lack of adequate infrastructure and services is typically associated with impoverished areas and sectors of the population.

The mosquito species Aedes aegypti lives in close association with humans, preferentially feeding on human blood and reproducing in human-created habitat [5]. It has been shown to be highly adapted to urban environments [6,11]. As hosts for the insect, humans face an increased likelihood of transmission with increasing population density [12]. Given $A$. aegypti ecology, rapid, unplanned urbanization has led to increased global incidence of dengue fever.

Previous studies have identified a large number of ecological, socioeconomic and demographic factors that affect the spatial and temporal extent of dengue outbreaks in Sri Lanka. Studies examining these variables have primarily considered different variable separately and has not clearly demonstrated predictive relationships. Dengue epidemiology could benefit from additional spatial information and predictive tools. This study examines the spatial and temporal relationships of dengue outbreaks in areas of northern Sri Lanka. The 
Citation: Anno S, Imaoka K, Tadono T, Igarashi T, Sivaganesh S, et al. (2014) Assessing the Temporal and Spatial Dynamics of the Dengue Epidemic in Northern Sri Lanka using Remote Sensing Data, GIS and Statistical Analysis. J Geophys Remote Sensing 3: 135. doi: 10.4172/2169-0049.1000135

Page 2 of 5

results can help clarify typical trajectories of the disease and inform surveillance and control strategies for this region.

\section{Materials and Methods}

\section{Study area}

Our study area in northern Sri Lanka ranged across twelve regions of health administration, referred to as Medical Office of Health $(\mathrm{MOH})$ divisions. Each $\mathrm{MOH}$ division consisted of a unique set of geographic features-including agricultural fields, forested areas, wetlands, grassland, urban areas, etc. Each division also has a unique socio-economic make-up. The climate in the region is tropical, with two monsoon seasons. These include the northeast monsoon (November to April), and the southwest monsoon (May to October).

\section{Dengue data}

The Sri Lankan $\mathrm{MOH}$ divisions provided monthly estimates of clinically confirmed dengue cases from January 2010 through December 2013. They also provided annual estimates of clinically confirmed dengue cases from 2007 through 2013.

\section{Rainfall, humidity and temperature}

Rainfall data were obtained and processed using the Global Satellite Mapping of Precipitation (GSMaP) freeware and the combined MWIR algorithm. Data were compiled from numerous satellites including TRMM TMI, Aqua AMSR-E, GCOM-W AMSR2, DMSP SSM/I, DMSP SSMIS, NOAA-19 AMSU, MetOp-A AMSU and GEO IR. The GSMap was drafted at the highest levels of precision and resolution (Temporal resolution: 1 hour, Spatial resolution: Grid latitudelongitude of 0.1 degrees) $[13,14]$. The near-real-time version (GSMaP_NRT) is published with an approximately 4-hour time-lag from the time of satellite observation, with the reanalysis version (GSMaP_MVK) available after reprocessing. The monthly average rainfall from January 2010 to December 2013 and the annual average rainfall from 2007 to 2013 were obtained by processing the GSMaP_MVK and GSMaP_NRT data for the study period, and converting processed data into TIFF image files for incorporation into the geographic information system (GIS) spatial model.

Humidity (total precipitable water, or vertically integrated water vapor amount) and temperature (surface temperature) data were acquired from the JAXA Satellite Monitoring for Environmental Studies (JASMES) data portal [15]. These geophysical parameters were acquired from Aqua/MODIS and Terra/MODIS data. Both the Terra and Aqua satellites include MODIS instruments. MODIS projects a swath of 2,330 km in width, and views the entire Earth's surface every one to two days. Its detectors measure 36 spectral bands between 0.405 and $14.385 \mu \mathrm{m}$, and it acquires data at three spatial resolutions: $250 \mathrm{~m}$, $500 \mathrm{~m}$ and $1,000 \mathrm{~m}$ [16]. We obtained the monthly averages from January 2010 to December 2013, and the annual averages from 2007 to 2013 by processing the RS data, and converting it into TIFF image files for integration into the GIS model.

\section{Land cover, including urbanization}

A paper survey map was digitized to generate detailed land cover data. The digital land cover map was also integrated into the GIS model.
The ALOS/AVNIR-2 dataset was used along with an unmix method to detect and map urbanization. The unmix method isolates the contribution of a specific material within a heterogeneous (mixed) pixel. For a given material, this method records pixel location and fraction of material present in the pixel. We designated eight material pixel fraction classes that report subpixel detections in material pixel fraction increments of 0.20 . Pixels determined to have material pixel fractions of $20-29 \%$ belong to class $0.20-0.29$, pixels with material pixel fractions of $90-100 \%$ belong to class $0.90-1.00$ and so forth.

\section{Population density}

We obtained annual population data for respective $\mathrm{MOH}$ divisions from 2007 to 2013 from the Regional Epidemiological Unit, Jaffna. Combining this information with the $\mathrm{MOH}$ division data for the area, we calculated the population density as a socio-economic and demographic factor. We also calculated average values from annual population density estimates at the same scale $(\mathrm{MOH}$ division level) to investigate the annual population density trends. These data were used for both spatial association analysis and spatial statistical analysis.

\section{Incidence rates}

To examine the spatial patterns of dengue occurrence, epidemic curves were produced by calculating the annual dengue incidence rate during a period from 2007 to 2013. Annual incidence rates for each $\mathrm{MOH}$ division were calculated from the number of annual confirmed dengue cases, divided by the total population-years and then multiplied by 10,000 . These rates were expressed as annual confirmed dengue cases per 10,000 individuals. The average annual incidence at the $\mathrm{MOH}$ division level was also calculated to determine overall trends in annual incidence. This was used for both spatial association analysis and spatial statistical analysis.

\section{Spatial analysis in GIS}

A polygon layer based on the twelve $\mathrm{MOH}$ divisions was integrated with TIFF image data on rainfall, humidity and temperature within the GIS model. The pixel (i.e., raster) size was changed from 0.05 to 0.01 to integrate layers and enable analysis of raster values within the polygons. We extracted raster value from pixels according to polygonal templates and then assigned summaries of raster values to each polygon. The results were tabulated in Excel.

The digital land cover map was also integrated into the GIS model. The polygon layer and the digital land cover map layer were overlaid. The land cover data within the polygons were summarized and tabulated in Excel. Similar methods were used to integrate raster data on urbanization with $\mathrm{MOH}$ spatial divisions. Thus the polygon layer attributes were also integrated with ecological, socio-economic and demographic data in Excel.

We also calculated the average annual values for rainfall, humidity and temperature within $\mathrm{MOH}$ divisions. This information allowed us to visualize variation in annual rainfall, humidity and temperature, and was used for both spatial association analysis and spatial statistical analysis.

\section{Temporal analysis}

To examine temporal patterns, we used monthly data on dengue cases, rainfall, humidity and temperature from January 2010 through December 2013. The moving average (MA) was calculated and 
Citation: Anno S, Imaoka K, Tadono T, Igarashi T, Sivaganesh S, et al. (2014) Assessing the Temporal and Spatial Dynamics of the Dengue Epidemic in Northern Sri Lanka using Remote Sensing Data, GIS and Statistical Analysis. J Geophys Remote Sensing 3: 135. doi: 10.4172/2169-0049.1000135

Page 3 of 5

graphed to examine temporal climate trends associated with dengue outbreaks. We also calculated average monthly values from monthly data on rainfall, humidity and temperature to determine trends and chi-square test inputs. The chi-square test was used to evaluate the randomness of variation in monthly estimates of dengue cases, rainfall, humidity and temperature during the study period. The significance levels were set at 0.05 .

\section{Spatial association analysis}

Univariate Local Indicators of Spatial Association (LISA) analysis (implemented in GeoDa) was used to measure the local spatial autocorrelation of dengue occurrence $[17,18]$. LISA statistics measure spatial dependence and evaluate the existence of local clusters within the spatial distribution of a given variable. They are based on a statistical index I, developed by Moran to measure the global spatial autocorrelation of the overall data clustering in a given spatial area under investigation [19]. Moran's I ranges from -1 (negative spatial autocorrelation) to 1 (complete spatial dependence), with 0 indicating an absence of spatial dependence (i.e., random distribution) [20].

Local spatial autocorrelation analysis was performed based on the Local Moran LISA statistics, which yield a measure of spatial autocorrelation for each individual location. LISA statistics revealed five categories of spatial autocorrelation presented on the cluster map legend: 1) not significant (i.e., areas that are not significant at the default pseudo-significance level of 0.05), 2) high-high (high values surrounded by high values), 3) low-low (low values surrounded by low values), 4) low-high (low values surrounded by high values) and 5) high-low (high values surrounded by low values) [21,22]. High-high and low-low represent positive spatial autocorrelation (i.e., association between areas of similar values), and high-low and low-high represent negative spatial correlation (i.e., association between areas of dissimilar values) [21,23]. A finding of significant clustering for $\mathrm{p}<0.05$ suggests that dengue incidence values are too similar in adjacent provinces to have occurred by chance, providing significant evidence for rejecting the null hypothesis of this pattern [23].

Assigning individuals to these four classes depends on the results of a statistical significance test. We used a test consisting of random Monte Carlo permutations among the sites located in the spatial lag, to compare the observed LISA results to those corresponding to the random permutations [17]. For this analysis, significance was calculated from 999 permutations, corresponding to a pseudo-p-value significance threshold of 0.01 and a confidence level of $99 \%$ [20].

\section{Spatial statistical analysis}

The chi-square test was used to test the spatial association between ecological, socio-economic and demographic factors and dengue cases. The ecological, socio-economic and demographic factors were categorized in two ways: 1) values above or below average values for a given factor or 2) presence or absence of factor depending on the factors. Threshold values for these factors were determined from average values or categorized according to their presence or absence within spatial units of the GIS model. The statistical significance levels were set at 0.05 .

Results from the chi-square test allowed us to compare differences in ecological, socio-economic and demographic factors between areas of significant high-high clustering (i.e., endemic areas) and the areas of significant low-high clustering (i.e., non-endemic areas predicted to be under the influence of other model variables), as identified in the univariate LISA analysis.

\section{Results}

\section{Results of temporal analysis}

Humidity levels tend to rise in early January in the study area, remain at elevated levels during the dry season, and then decline with increasing rainfall in early September. These changes accelerate at lower temperatures. Distributions of monthly dengue cases show a compelling degree of variation in step with weather variables. Dengue case specifically tended to increase after exponential increases or decreases in rainfall (Figure 1). The chi-square test results affirmed the significance of the relationship. We observed significant monthly covariance between dengue cases and rainfall $(\mathrm{p}<0.01)$, whereas dengue cases did not appear to vary with humidity or temperature variables.

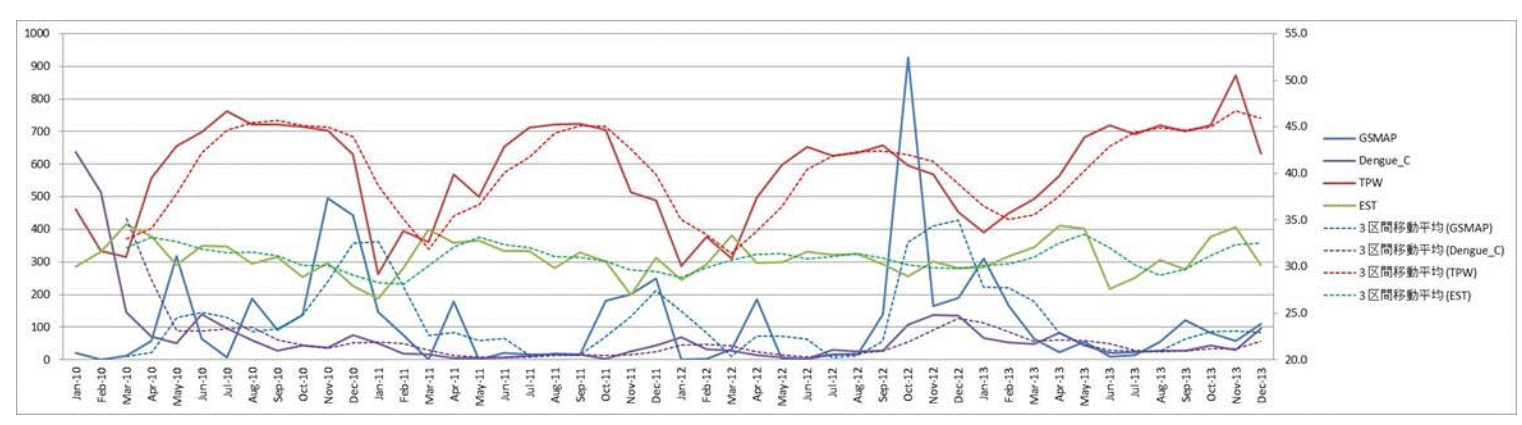

Figure 1: The temporal trends for monthly dengue cases, rainfall, humidity and temperature. Monthly dengue cases in the northern region of Sri Lanka from January 2010 to December 2013 (purple solid line); the average monthly rainfall in mm (blue solid line); the average monthly humidity in ${ }^{\circ} \mathrm{C}$ (red solid line); and the average monthly temperature in ${ }^{\circ} \mathrm{C}$ (green solid line). The dashed lines show three-month moving averages of dengue cases (purple), rainfall (blue), humidity (red) and temperature (green). 
Citation: Anno S, Imaoka K, Tadono T, Igarashi T, Sivaganesh S, et al. (2014) Assessing the Temporal and Spatial Dynamics of the Dengue Epidemic in Northern Sri Lanka using Remote Sensing Data, GIS and Statistical Analysis. J Geophys Remote Sensing 3: 135. doi: 10.4172/2169-0049.1000135

Page 4 of 5

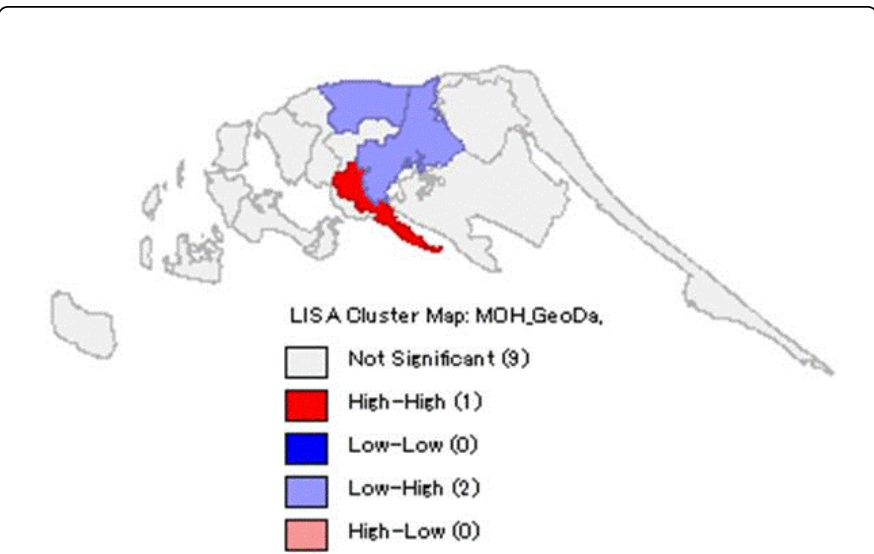

Figure 2: LISA cluster map.

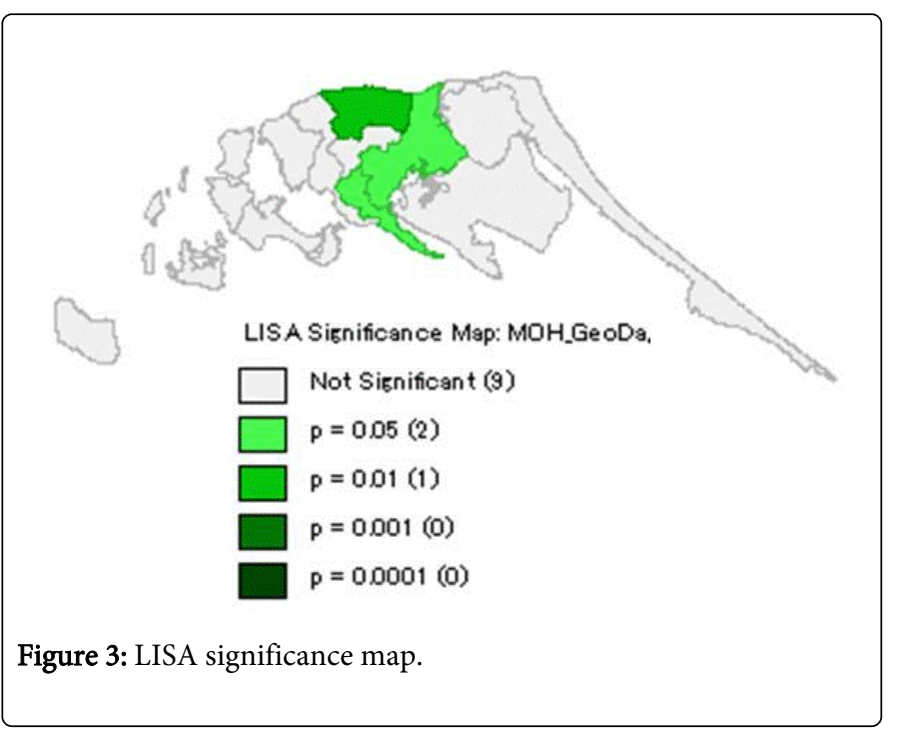

\section{Results of spatial association analysis}

Figures 2 and 3 show the LISA cluster and significance maps (respectively) of average values based on annual dengue incidences. The LISA cluster map shows two types of geographical clustering (high-high and low-high). The most significant area of high-high clustering (average annual dengue incidences) accounted for $8.3 \%$ of observed clusters and occurred in the Nallur $\mathrm{MOH}$ division. The most significant area of low-high clustering accounted for $16.7 \%$ of observed clusters and occurred in Kopay and Tellipallai MOH divisions. The Moran's I statistic of -0.08 indicated only random spatial variation.

\section{Results of spatial statistical analysis}

The spatial statistical analysis revealed that dengue occurrence correlates with ecological, socio-economic and demographic factors. Significantly more dengue cases were observed in $\mathrm{MOH}$ divisions $(66.7 \%)$ with average annual rainfall of $>1353 \mathrm{~mm}$ compared to in those with average annual rainfall of $<1353 \mathrm{~mm}\left(\chi^{2}=112.8 ; \mathrm{p}<0.01\right)$. Correspondingly, we also observed significantly more dengue cases in $\mathrm{MOH}$ divisions (66.7\%) with average annual humidity of $>39.62 \mathrm{~mm}$ compared to those with average annual humidity of $<39.62 \mathrm{~mm}$ $\left(\chi^{2}=55.6 ; \mathrm{p}<0.01\right)$. Moreover, significantly more dengue cases occurred in $\mathrm{MOH}$ divisions (58.3\%) with an average annual temperature $>31.2^{\circ} \mathrm{C}$ compared to those with an average annual temperature of $<31.2^{\circ} \mathrm{C}\left(\chi^{2}=104.7 ; \mathrm{p}<0.01\right)$ (Table 1$)$.

Dengue occurrence was also significantly associated with the presence or absence of urbanized areas $\left(\chi^{2}=264.7 ; \mathrm{p}<0.01\right)$. Urbanization within $\mathrm{MOH}$ divisions (33.3\%) covaried with dengue occurrence. Significantly more dengue cases occurred in $\mathrm{MOH}$ divisions $(50.0 \%)$ that had an urbanization ratio of $>18 \%$ within the $\mathrm{MOH}$ division area, relative to those with urbanization ratios of $<18 \%$ $\left(\chi^{2}=40.7 ; \mathrm{p}<0.01\right) . \mathrm{MOH}$ divisions with a population density of $>1150$ (33.3\%) hosted a higher number of dengue cases relative to those with population densities of $<1150\left(\chi^{2}=347.2 ; \mathrm{p}<0.01\right)$.

\begin{tabular}{|c|c|c|c|}
\hline \multicolumn{2}{|l|}{ Factors } & $\mathrm{N}(\%)$ & $p\left(x^{2}\right)$ \\
\hline \multirow[t]{2}{*}{ Rainfall } & $>1353 \mathrm{~mm}$ & $8(66.7 \%)$ & \multirow[t]{2}{*}{$p<0.01(112.8)$} \\
\hline & $<1353 \mathrm{~mm}$ & $4(33.3 \%)$ & \\
\hline \multirow[t]{2}{*}{ Humidity } & $>39.62 \mathrm{~mm}$ & $8(66.7 \%)$ & \multirow[t]{2}{*}{$p<0.01(55.6)$} \\
\hline & $<39.62 \mathrm{~mm}$ & $4(33.3 \%)$ & \\
\hline \multirow[t]{2}{*}{ Temperature } & $>31.2^{\circ} \mathrm{C}$ & $7(58.3 \%)$ & \multirow[t]{2}{*}{$p<0.01(104.7)$} \\
\hline & $<31.2^{\circ} \mathrm{C}$ & $5(41.7 \%)$ & \\
\hline \multirow[t]{2}{*}{ Built-up area } & Presence & $4(33.3 \%)$ & \multirow[t]{2}{*}{$p<0.01(264.7)$} \\
\hline & Absence & $8(66.7 \%)$ & \\
\hline \multirow[t]{2}{*}{ Urbanization } & $>18 \%$ & $6(50.0 \%)$ & \multirow[t]{2}{*}{$p<0.01(40.7)$} \\
\hline & $<18 \%$ & $6(50.0 \%)$ & \\
\hline \multirow[t]{2}{*}{ Pop. Density } & $>1150$ & $4(33.3 \%)$ & \multirow[t]{2}{*}{$p<0.01(347.2)$} \\
\hline & $<1150$ & $8(66.7 \%)$ & \\
\hline
\end{tabular}

Table 1: Results of spatial statistical analysis.

The chi-square test results showed the Nallur $\mathrm{MOH}$ division to be a high-high clustering area, with urbanized land area and a relatively high population density. The Kopay and Tellipallai $\mathrm{MOH}$ divisions represented low-high clustering areas having a smaller relative proportion of urbanized area and lower population densities. These results indicate significant differences in urbanization and population density between high-high clustering areas and low-high clustering areas. Urbanization and higher population density could thus influence dengue occurrence.

\section{Discussion}

Our results showed that dengue outbreaks covary with rainfall, humidity, temperature, and urbanization and population density. Our analyses also quantify the degree to which these factors influence dengue occurrence. The results of spatial association analysis showed that even though Nallur, Koppay and Tellipallai $\mathrm{MOH}$ divisions cluster together, Nallur represented an endemic area, whereas Kopay and Tellipallai do not. Variation in dengue cases corresponds to variation in urbanization and population density between these endemic and non-endemic areas. Urbanization and higher population density could thus influence dengue occurrence. This interpretation is consistent with ecology and behaviour of the vector (i.e., Aedes 
Citation: Anno S, Imaoka K, Tadono T, Igarashi T, Sivaganesh S, et al. (2014) Assessing the Temporal and Spatial Dynamics of the Dengue Epidemic in Northern Sri Lanka using Remote Sensing Data, GIS and Statistical Analysis. J Geophys Remote Sensing 3: 135. doi: 10.4172/2169-0049.1000135

Page 5 of 5

aegypti), which is an opportunistic breeder, highly adapted to urban and specifically residential environments.

Data concerning urbanization trends were obtained from the digital land cover map, and not the RS data in the present study. Future research efforts should be made to determine urbanization trends from RS data to verify and refine our results and interpretations.

\section{Conclusion}

Dengue occurrence within northern Sri Lanka is spatially and temporally heterogeneous. As such it requires integration of statistical analysis and GIS. Advances in remote sensing (RS) and the increasing availability of RS data can be used to refine epidemeological models of re-emerging diseases such as dengue fever. Spatial and temporal analysis of cases of dengue fever showed that rainfall, humidity, temperature, urbanization and higher population density are predictive variables in the disease's occurrence in northern Sri Lanka. Further research must focus on the whole island to improve the accuracy and scope of spatio-temporal models. An integrated spatiotemporal prediction model that uses ecological, socio-economic and demographic factors could substantially improve risk assessment, prevention and control of dengue outbreaks.

\section{References}

1. Tam CC, Tissera H, de Silva AM, De Silva AD, Margolis HS, et al. (2013) Estimates of dengue force of infection in children in Colombo, Sri Lanka. PLoS Negl Trop Dis 7: e2259.

2. Kanakaratne N, Wahala WM, Messer WB, Tissera HA, Shahani A, et al. (2009) Severe dengue epidemics in Sri Lanka, 2003-2006. Emerg Infect Dis 15: 192-199.

3. WHO South East Asia Regional Office (2011) Comprehensive Guidelines for Prevention and Control of Dengue and Dengue Haemorrhagic Fever - Revised and Expanded Edition, New Delhi.

4. Canyon DV, Hii JL, Müller R (1999) Adaptation of Aedes aegypti (Diptera: Culicidae) oviposition behavior in response to humidity and diet. J Insect Physiol 45: 959-964.

5. Gubler DJ (1997) Dengue and Dengue Hemorrhagic Fever: Its History and Resurgence as a Global Public Health Problem, In: Gubler DJ, and Kuno G, eds., Dengue and Dengue Hemorrhagic Fever. CAB International, Wallingford, United Kingdom,1-22.
6. Rodhain F, Rosen L (1997) Mosquito vectors and dengue virus-vector relationships, In: Gubler DJ and Kuno G eds, Dengue and Dengue Hemorrhagic Fever. CAB International, Wallingford, United Kingdom.

7. Cheah WL, Chang MS, Wang YC (2006) Spatial, environmental and entomological risk factors analysis on a rural dengue outbreak in Lundu District in Sarawak, Malaysia. Trop Biomed 23: 85-96.

8. Ooi EE, Gubler DJ (2009) Dengue in Southeast Asia: epidemiological characteristics and strategic challenges in disease prevention. Cad Saude Publica 25 Suppl 1: 115-124.

9. Tran HP, Adams J, Jeffery JA, Nguyen YT, and Vu NS (2010) Householder perspectives and preferences on water storage and use, with references to dengue, in the Mekong Delta, southern Vietnam. International Health 2: 136-142.

10. Gubler DJ (2011) Dengue, Urbanization and Globalization: The Unholy Trinity of the 21(st) Century. Trop Med Health 39: 3-11.

11. WHO (2012) Dengue and Severe Dengue, Fact Sheet Nu117.

12. Gubler DJ (1998) Dengue and dengue hemorrhagic fever. Clin Microbiol Rev 11: 480-496.

13. JAXA (2014) JAXA Global Rainfall Watch.

14. RESTEC (2014) Global Satellite Mapping of Precipitation (GSMaP).

15. JAXA (2014b) JAXA Satellite Monitoring for Environmental Studies (JASMES).

16. NASA (2014) National Aeronautics and Space Administration.

17. Anselin L (1995) Local indicators of spatial association-LISA. Geographical Analysis 27: 93-116.

18. Anselin L, Syabri I, Kho Y (2006) GeoDa: An introduction to spatial data analysis. Geographical Analysis 38: 5-22.

19. MORAN PA (1950) Notes on continuous stochastic phenomena. Biometrika 37: 17-23.

20. Guessous I, Joost S, Jeannot E, Theler JM and Mahler P (2014) GIRAPH Group. A comparison of the spatial dependence of body mass index among adults and children in a Swiss general population. Nutrition Diabetes 4: e111.

21. Anselin L (2003) $\mathrm{GeoDa}^{\mathrm{Tm}} 0.9$ User's Guide. University of Illinois, Urbana-Champaign, Urbana, Illinois.

22. Singh A, Pathak PK, Chauhan RK, Pan W (2011) Infant and child mortality in India in the last two decades: a geospatial analysis. PLoS One 6: 26856.

23. Martinez AN, Mobley LR, Lorvick J, Novak SP, Lopez A, et al. (2014) Spatial analysis of HIV positive injection drug users in San Francisco, 1987 to 2005. Int J Environ Res Public Health 11: 3937-3955. 